\title{
Tsafon
}

Revue d'études juives du Nord

$81 \mid 2021$

Des synagogues à travers les âges Lieux de prières, lieux d'études et autres fonctions

\section{La synagogue La Victoire}

monument emblématique du franco-judaïsme et des contradictions contemporaines du judaïsme français

\section{Claude Nataf}

\section{OpenEdition}

\section{Journals}

Édition électronique

URL : https://journals.openedition.org/tsafon/3764

DOI : $10.4000 /$ tsafon.3764

ISSN : 2609-6420

Éditeur

Association Jean-Marie Delmaire

Édition imprimée

Date de publication : 1 juillet 2021

Pagination : 73-88

ISSN : 1149-6630

Référence électronique

Claude Nataf, « La synagogue La Victoire », Tsafon [En ligne], 81 | 2021, mis en ligne le 01 juillet 2021 consulté le 15 septembre 2021. URL : http://journals.openedition.org/tsafon/3764 ; DOI : https:// doi.org/10.4000/tsafon.3764 


\title{
La synagogue La Victoire,
}

\author{
monument emblématique du franco-judaïsme \\ et des contradictions contemporaines du judaïsme français
}

\author{
Claude Nataf*
}

La Révolution française a émancipé les Juifs de France. Elle leur a donné la liberté de circuler, de s'établir à leur convenance à l'intérieur de l'Hexagone, de pratiquer leur culte, de prétendre à tous les emplois publics et privés, en mettant fin à toutes les discriminations et exceptions qu'ils avaient pu connaître sous l'Ancien Régime. Cette liberté et cette égalité reconnues aux individus ne consacraient cependant pas une égalité de droit et de fait entre le culte israélite et les cultes catholique et protestant. Aussi les dirigeants du judaïsme français n'ont eu de cesse, depuis la création de l'institution consistoriale par Napoléon, de donner à la synagogue une place comparable à celle des églises chrétiennes dans le paysage français. Cet effort continu préside-à la création de la synagogue de la rue de La Victoire conçue sous le Second Empire et inaugurée dans les premières années de la Troisième République ${ }^{1}$.

Symbole du judaïsme français, elle se confond, depuis 1874, avec son histoire et a été le cadre de ses moments les plus dramatiques et de son intégration à la France. Elle subit aussi depuis la fin de la Seconde Guerre mondiale les difficultés internes et externes de la communauté juive de France dont la composition s'est profondément modifiée depuis 1874 et qui est confrontée à un renouveau religieux refusant le modèle consistorial,

*Historien, vice-président de la Commission française des Archives juives.

${ }^{1}$ Sur l'histoire de la synagogue de La Victoire, voir Jacques Canet et Claude Nataf (dir.), La synagogue de la Victoire 150 ans du judaïsme français, Paris, Porte-plume éditions, 2017. 
à l'existence de l'État d'Israël et à un antisémitisme différent de l'antisémitisme traditionnel qui interpelle les nouvelles générations sur leur place dans la Nation.

\section{La genèse d'une synagogue}

Le décret napoléonien du 17 mars 1808 dispose qu'il sera établi une synagogue dans chaque département comprenant 2.000 israélites. Paris fait cependant exception à la règle puisque deux synagogues y sont créées, moins en raison du nombre de Juifs de la ville que pour tenir compte des différences de rites entre les personnes originaires des régions de l'est de la France et ceux du sud-ouest et des anciens États pontificaux.

Ces deux synagogues sont situées dans des voies secondaires, rue Saint-Avoye (actuelle rue du Temple) et rue de Chaume, ne se distinguent par aucun signe extérieur, sont exiguës et ne suffisent pas aux besoins de la population parisienne. Si les Juifs de France sont égaux en droit à leurs concitoyens, la synagogue est encore située dans de petites rues, à l'abri des regards. Contrairement aux cultes catholique et protestant, les dépenses relatives au culte juif, y compris le salaire des rabbins, ne sont pas prises en charge par l'État.

La population juive parisienne croissant de façon continue, le besoin d'une grande synagogue se fait sentir dès les premières années de la Restauration. Les notables souhaitent l'installer dans un bâtiment imposant et dans un quartier à forte population juive, ce qui les conduit à repousser la proposition du baron James de Rothschild de leur offrir un terrain situé dans l'actuelle rue Chauchat. Louis XVIII, sur leur demande, les autorise à acquérir un terrain rue Notre-Dame-de-Nazareth en plein cœur d'un quartier très commerçant mais ils refusent toute aide de l'État ou de la ville de Paris en se fondant sur les dispositions du décret napoléonien. Le consistoire fait donc appel à des dons de fidèles et recourt à un emprunt pour financer la construction.

La synagogue est inaugurée en 1822. Si elle n'est pas implantée dans une ruelle mais dans un quartier animé au cœur de la cité, elle est d'une grande discrétion à l'extérieur. Il n'y a pas un seul symbole juif sur la façade qui permette de distinguer l'immeuble. L'entrée sur la rue débouche sur une cour intérieure et c'est à l'intérieur de cette cour que se trouve la synagogue, qui n'est donc pas visible de l'extérieur. L'intérieur de la synagogue ressemble à un temple néoclassique. Un petit bâtiment annexe ouvrant sur la rue Neuve Saint-Laurent (actuelle rue du Vertbois) 
abrite une seconde synagogue plus petite, réservée au rite hispanoportugais. La construction ayant été menée à l'économie, le bâtiment se dégrade assez vite et des risques d'effondrement sont signalés à différentes reprises par les services préfectoraux qui menacent de le fermer. Le consistoire décide donc de le démolir et de procéder à sa reconstruction. La synagogue reconstruite est inaugurée en 1852. Pour la première fois, à Paris, elle s'affirme comme telle vis-à-vis de l'extérieur. À l'intérieur on s'est inspiré du modèle des cathédrales françaises. Mais cette synagogue reconstruite et agrandie s'avère rapidement insuffisante aux besoins du judaïsme parisien ${ }^{2}$.

La reconstruction de 1852 coïncide avec les grands travaux entrepris à Paris par le préfet Haussmann sur ordre de Napoléon III qui veut créer dans le centre de Paris un quartier prestigieux abritant les sièges de grandes sociétés financières et industrielles, de l'Opéra, des théâtres, des commerces de luxe, des lieux où l'on s'amuse et des demeures d'une bourgeoisie triomphante faisant pendant à celles du Faubourg SaintGermain, foyer d'opposition à l'Empire. C'est pourquoi le baron Gustave de Rothschild a immédiatement l'idée de proposer au consistoire et à Haussmann l'édification d'une synagogue au cœur de ce quartier en gestation $^{3}$. Le consistoire a signalé la nécessité de créer une seconde synagogue mais il souhaite l'implanter dans le Marais où réside une importante population juive. Haussmann, pour sa part, est très réticent à l'édification d'une synagogue dans le futur quartier destiné à être la vitrine de Paris. Il appuie l'idée d'une nouvelle construction dans le quartier du Marais tout en précisant que la ville de Paris ne pourra apporter son concours financier.

Mais Gustave de Rothschild ne renonce pas à son souhait de la présence d'une synagogue dans le quartier destiné à être celui de l'élite car il voit là un moyen d'assurer au judaïsme une égalité de traitement avec les autres cultes et sa reconnaissance comme partie intégrante de la France. Devenu président du consistoire de Paris en 1858, il reprend ses démarches

\footnotetext{
${ }^{2}$ En 1810, on recense environ 2.700 Juifs à Paris. En 1820, on en compte 7.000 environ. La chute de l'Empire entraine l'arrivée en France de Juifs habitant les territoires détachés de la France par le traité de Paris et qui ne veulent pas perdre le statut de liberté et d'égalité qu'ils avaient connu comme citoyens de l'Empire. En 1857, le nombre de Juifs parisiens est de 13.000 avec l'installation de Juifs d'Alsace-Lorraine attirés par l'expansion de la capitale et des Juifs de Russie et d'Orient fuyant les persécutions et espérant trouver en France un avenir meilleur. (D'après Roger Berg, Histoire des Juifs à Paris de Chilpéric à Jacques Chirac, Paris, éditions du Cerf, 1997).

${ }^{3}$ Gustave de Rothschild (1829-1911), fils cadet du baron James est alors membre du consistoire de Paris dont il deviendra président en 1858.
} 
auprès d'Haussmann et affirme sa volonté d'édifier concomitamment une synagogue dans le quartier du Marais et une autre dans le IX ${ }^{\mathrm{e}}$ arrondissement. Il propose même un terrain situé rue de Châteaudun. Haussmann refuse car il l'estime trop proche de l'église Notre-Dame-deLorette et que, dans certains cas, il pourrait résulter des inconvénients de la proximité de ces deux temples ${ }^{4}$. Gustave de Rothschild intervient auprès de Napoléon III et c'est ce dernier qui donne pour instructions à Haussmann de "satisfaire aux légitimes exigences du culte israélite ${ }^{5}$. Cette intervention est décisive et lève les réticences d'Haussmann. La ville de Paris cède au consistoire un terrain situé 44 rue de La Victoire où s'élevait un hôtel particulier acheté, en 1802, par Napoléon et Joséphine pour constituer la dot de Louis Bonaparte et d'Hortense de Beauharnais, et revendu par ces derniers à un financier avant d'être acheté par la ville de Paris pour les besoins des travaux du nouveau quartier. La ville de Paris consent au consistoire un emprunt de 2.000.000 de francs, remboursable en 75 années mais, l'achat du terrain et la construction représentant un investissement beaucoup plus important, le consistoire fait également appel à la générosité des fidèles. Une bonne partie des dons émane d'ailleurs des membres de la famille Rothschild.

Les travaux de la synagogue débutent en 1865. Ils sont interrompus par la guerre de 1870 puis l'épisode de la Commune de Paris et reprennent en 1871. Elle est inaugurée lors des fêtes de Tichri en 1874 et ouverte au culte public en 1875. On voit donc que l'édification de la synagogue de la rue de La Victoire procède d'une volonté politique au sens noble : marquer l'intégration des Juifs de France dans la Nation et l'égalité entre tous les cultes. Cette volonté se traduit aussi dans le projet architectural lui-même : édifier un lieu de culte monumental qui ne se distingue pas des lieux de cultes chrétiens et s'insère dans l'urbanisme haussmannien.

La façade de la synagogue s'inspire du modèle byzantin pour rappeler l'origine orientale du judaïsme; elle est marquée par la symbolique des Tables de la Loi qui surmontent le fronton ainsi que par la rose centrale au motif de l'étoile à six branches encadrée par des versets en hébreu. À l'intérieur, le vestibule franchi, le plan basilical des cathédrales témoigne de l'intégration des Juifs à la Nation française, y compris la mosaïque de pavements en forme de croix latine, le chœur, et les dimensions inédites pour une synagogue qui sont proches de celles

\footnotetext{
${ }^{4}$ Archives de l'Association consistoriale israélite de Paris (ci-après ACIP), AA5, séance du 5 novembre 1860, p. 219.

${ }^{5}$ Archives Nationales (ci-après AN), série F 19/11119.
} 
d'une grande église médiévale. Ce modèle inspire, dans les années suivantes, d'autres synagogues comme celles de la rue des Tournelles et de la rue Buffault pour ne citer que les parisiennes ${ }^{6}$.

La conception architecturale de la synagogue est la manifestation de la volonté, de la part des notables consistoriaux de s'intégrer dans la Nation.

\section{La synagogue de La Victoire symbole du franco-judaïsme}

L'ouverture de la synagogue au culte public est concomitante au développement du quartier prestigieux dont l'aménagement a commencé sous le Second Empire : quartier des grandes sociétés dont leurs dirigeants participent à des réunions feutrées de conseils d'administration, quartier où l'animation commerçante n'est pas de mise mais est remplacée, le soir, par l'ouverture des grands restaurants, des théâtres et de l'Opéra. La haute bourgeoisie juive y a donc tout naturellement aussi son lieu de culte que ses membres fréquentent selon leur degré de pratique religieuse mais où elle célèbre ses cérémonies familiales et surtout les mariages de ses enfants. C'est à l'occasion de ces événements mondains que les non-Juifs découvrent la synagogue.

Cyril Grange a cité le compte rendu, paru dans Le Figaro, du mariage de Charlotte-Béatrix de Rothschild, fille du président du consistoire central Alphonse de Rothschild, avec Maurice Ephrussi à La Victoire: «À cette occasion la demeure de l'Éternel déploie un luxe inusité d'harmonies, de fleurs, de tentures et de lumières. Les gens de marque franchissent le parvis. Ce n'est plus une noce : c'est un événement dont le retentissement est universel $\gg{ }^{7}$.

Destinée à être un lieu de culte, la synagogue de la rue de La Victoire rompt avec la notion ancienne de bet haknesset "maison d'assemblée » que recouvre encore son nom français de synagogue par référence au grec sunagogê «assemblée, réunion ». Mais elle est aussi le symbole d'un modèle social et religieux à la fois que les historiens ont appelé le francojudaïsme. Celui-ci repose sur l'appréhension du judaïsme comme une

\footnotetext{
${ }^{6}$ Sur l'architecture de la synagogue, cf. l'importante contribution de Nelly Singer « La Victoire : une nouvelle synagogue consistoriale dans le Paris haussmannien »dans Jacques Canet et Claude Nataf (dir.), op. cit, p. 32-53, également Dominique Jarrassé, L'âge d'or des synagogues, Paris, Herscher, 1991 et Idem, Guide du patrimoine juif parisien, Paris, Éditions Parigramme-Compagnie parisienne du Livre, 2003.

${ }^{7}$ Cyril Grange, « La synagogue de la rue de La Victoire, temple de la grande bourgeoisie parisienne », dans Jacques Canet et Claude Nataf (dir.), op. cit, p. 152-155.
} 
religion et rien d'autre. Par conséquent, le judaïsme relève exclusivement de la sphère privée des individus qui pratiquent cette religion.

L'institution consistoriale se donne pour mission de favoriser l'assimilation totale des Juifs à la Nation française par l'école et par l'armée. Mais la synagogue a aussi son rôle à jouer dans ce développement du franco-judaïsme. Elle a, bien sûr, la mission d'être le lieu de culte des citoyens de confession juive. Mais parce que La Victoire est par sa situation géographique la synagogue par excellence des notables et des dirigeants consistoriaux, parce qu'elle est le siège du grand rabbinat de Paris et du grand rabbinat de France, elle a un rôle particulier dans le paysage communautaire. C'est le lieu où le grand rabbin de France délivre ses messages spirituels de portée nationale. C'est le lieu encore des grandes célébrations nationales où, à l'unisson des autres cultes catholique et protestant, les Juifs de France rendent hommage à des personnalités nationales comme les présidents de la République Sadi Carnot (1894) et Félix Faure (1899) décédés au cours de leur mandat ou manifestent leur attachement à de grands événements patriotiques. L'histoire de la synagogue de la rue de La Victoire est inséparable non seulement de l'histoire du judaïsme français depuis 1875 mais aussi de l'histoire de France depuis cette date.

Sa construction exprime les sentiments de la bourgeoisie triomphante dans la France contemporaine. Plus tard, les querelles et les doutes liés à l'Affaire Dreyfus qui aboutissent en 1905 à la Loi de séparation des Églises et de l'État ont sans nul doute des échos profonds en son $\operatorname{sein}^{8}$.

À partir de 1914, La Victoire devient le lieu des grandes manifestations patriotiques du judaïsme français puis de l'Union sacrée à l'heure où Maurice Barrès se repent de son antisémitisme de 1894 et reconnait au judaïsme sa place dans les familles spirituelles de la France 9 . Le 29 mai 1916, le Président de la République Raymond Poincaré y assiste à une cérémonie d'hommage aux morts pour la France comme il l'avait fait à la Sainte-Chapelle ou au temple protestant de l'Oratoire. Ornée de

\footnotetext{
${ }^{8}$ Les beaux-parents d'Alfred Dreyfus (Hadamard) étaient des fidèles de La Victoire. C'est d'ailleurs dans cette synagogue qu'avait été célébré le 21 avril 1890, par Zadoc Kahn alors grand rabbin de Paris, le mariage du capitaine Dreyfus et de Lucie Hadamard et c'est à La Victoire que, bouleversé par les cris antisémites entendus lors de la dégradation d'Alfred Dreyfus, Théodore Herzl tente d'intéresser le grand rabbin de France et les dirigeants du judaïsme français à sa future publication L'Etat des Juifs qui envisage la création d'un État pour les Juifs.

${ }^{9}$ Maurice Barrès, Les Diverses Familles spirituelles de la France, Paris, édition ÉmilePaul Frères, 1917.
} 
drapeaux tricolores, la synagogue reçoit, pour la première fois dans 1'histoire du judaïsme français, la visite du chef de l'État ${ }^{10}$. Le journal consistorial L'Univers israélite du 9 juin 1916 écrit : «C'est au tour de la synagogue de La Victoire symbole du judaïsme français d'avoir l'honneur de recevoir le chef de l'État ${ }^{11}$. Après l'Armistice, c'est la cour de la synagogue de La Victoire qui est choisie pour abriter le Monument aux Morts pour la France qui est inauguré en 1923 en présence des autorités officielles. Face à ce monument, une plaque est apposée à la mémoire de Charles Péguy mort pour la France en 1914, à la demande de sa veuve, en hommage à l'action constante de ce dernier contre l'antisémitisme et en faveur de la révision du procès Dreyfus.

La synagogue de La Victoire devient dès lors le lieu des cérémonies commémoratives organisées par les associations d'anciens combattants, en particulier par Les Croix de Feu, et par les pouvoirs publics à l'instar de celles qui sont organisées dans les églises et les temples pour commémorer l'armistice le 11 novembre 1918. Ces cérémonies marquent l'âge d'or du franco-judaïsme et le triomphe d'un modèle français qui a su, tout en maintenant la pratique religieuse, faire reconnaître sa place dans la République. Chaque célébration s'achève par le chant de La Marseillaise après un discours rabbinique qui évoque le sacrifice des soldats.

En septembre 1939, lors de la déclaration de guerre c'est à la synagogue de La Victoire que le grand rabbin Isaïe Schwartz, récemment élu grand rabbin de France, bénit les armées françaises et affirme sa foi dans le destin de la France des Droits de l'homme.

\section{L'ère des doutes}

En juin 1940, le consistoire de Paris instruit de l'attitude des nazis, lors de leur entrée à Vienne puis à Prague, à l'égard de la communauté juive avait décidé que les membres du corps rabbinique non mobilisé seraient évacués en province.

Cependant un groupe de fidèles se retrouve à la synagogue de La Victoire pour y célébrer les offices du soir et décide d'assurer la permanence du culte, comme une forme de résistance spirituelle à l'entrée

\footnotetext{
${ }^{10}$ En 1782, le futur Louis XVIII alors comte de Provence, à l'occasion d'un voyage en Lorraine où il représentait son frère Louis XVI, avait été reçu à un office d'hommage au roi de France, à la synagogue de Metz.

${ }^{11}$ Cf. Philippe Landau, « 29 mai 1916 : visite officielle de Raymond Poincaré à La Victoire », dans Jacques Canet et Claude Nataf (dir.), op. cit, p. 176-177.
} 
des forces allemandes dans la capitale ${ }^{12}$. Le grand rabbin de Paris, Julien Weill, évacué en zone sud avec l'ensemble du rabbinat parisien, franchit clandestinement la ligne de démarcation pour regagner Paris. Persuadé que son devoir est d'être auprès de sa communauté dans les temps d'épreuves, il réactive le culte juif à Paris car les besoins d'une population en souffrance sont immenses. Le journaliste Jacques Bielinky note dans son journal, à la date du 20 juillet 1940, la présence de beaucoup de monde à la synagogue de La Victoire et constate que l'on récite comme d'usage la prière pour la France ${ }^{13}$. Pendant toute l'occupation allemande, le grand rabbin Julien Weill, malgré les menaces, les intimidations, les persécutions des SS, maintient ouverte la synagogue de la rue de La Victoire et y célèbre les offices quotidiens, les offices du shabbat et des grandes fêtes. Il célèbre également des mariages et des bar-mitsva.

Dans la nuit du 2 au 3 octobre 1941 la synagogue de La Victoire, comme d'autres synagogues parisiennes, est endommagée par une bombe placée à l'intérieur du péristyle. Un second attentat, qui est le fait de militants du Parti Populaire Français de Doriot, a lieu en 1942 à l'intérieur de l'édifice. L'appartement de Julien Weill est plusieurs fois envahi et saccagé. En raison des dégâts causés à la synagogue, les offices se déroulent dans l'oratoire pendant les jours qui suivent la libération de Paris. De nombreux militaires américains y assistent et, plus tard, ils encouragent l'aide du judaïsme américain à la remise en état de la synagogue. À la demande du gouvernement provisoire une cérémonie d'action de grâces pour la libération de Paris est organisée à la synagogue de La Victoire dans la continuité de son rôle d'avant-guerre. Toutefois le grand rabbin Julien Weill fait remarquer qu'il est impossible aux Juifs de Paris de se réjouir pleinement alors qu'ils sont incertains quant au sort de leurs coreligionnaires déportés. Une nouvelle problématique se pose désormais au judaïsme français. Après avoir été considérés comme des parias par le gouvernement de Vichy, internés et livrés à l'occupant allemand, les Juifs de France ont-ils désormais une autre place dans la Nation et leur fidélité traditionnelle à la patrie sacralisée par la devise consistoriale « Religion et Patrie » est-elle toujours de mise ?

Voulant renouer avec le cours suivi avant la Seconde Guerre mondiale, le consistoire de Paris décide d'apposer une plaque de bronze

\footnotetext{
${ }^{12}$ Voir Claude Nataf, «La Victoire pendant l'occupation allemande : juin 1940-août $1944 »$, dans Jacques Canet et Claude Nataf (dir.), op. cit, p. 204-215.

${ }^{13}$ Jacques Bielinky, Un journaliste juif sous l'occupation, établi, présenté et annoté par Renée Poznanski, Paris, Cerf, 1992.
} 
dans la synagogue de La Victoire, destinée à rappeler « éternellement la barbarie nazie » et ses victimes innombrables sans réserver ce monument du souvenir aux seuls morts au champ d'honneur, comme c'était le cas pour la Première Guerre mondiale, mais en y associant tous les morts en déportation. Le grand rabbin de France, Isaïe Schwartz, fait remarquer la distinction qui s'impose entre les déportés pour faits de résistance et les déportés parce que juifs ! Mais malgré les réserves du rabbinat le président du consistoire de Paris, Georges Wormser ${ }^{14}$, use de tout son poids pour ne pas faire la distinction. Sur sa proposition, un monument dédié à la mémoire des combattants de la guerre et de la résistance et aux martyrs de la déportation est érigé dans le péristyle de la synagogue. En raison de difficultés financières et architecturales, il n'est terminé qu'en 1948, il est inauguré le 27 février 1949 par le Président de la République, Vincent Auriol, renouvelant ainsi la visite de Raymond Poincaré à la synagogue en 1916. Georges Wormser, dans un discours très protocolaire, unit le martyrologe juif aux sacrifices de tous les Français : « Par-delà nos propres morts et confondus avec eux, nous entendons honorer aujourd'hui sans distinction de confession ou d'appartenance tous ceux qui sont tombés pour elle [la France], sur les champs de bataille, dans les maquis, en déportation, dans les camps de travail ou dans les prisons de la répression ${ }^{15}$. Ce refus d'un martyrologe juif spécifique et la visite de Vincent Auriol semblent marquer le retour du franco-judaïsme dont la synagogue parisienne redevient l'emblème et confirment la reconnaissance du retour du judaïsme français dans la Nation. Cette vision doit cependant être nuancée car des voix discordantes se démarquent de la position consistoriale, notamment par la voix du rabbinat.

La création de l'État d'Israël remet en question ce rôle emblématique $\mathrm{du}$ franco-judaïsme tenu par la synagogue de la rue de La Victoire. À cette occasion, le grand rabbin Isaïe Schwartz veut y faire célébrer une cérémonie d'action de grâces. Le président Georges Wormser s'y oppose puisque l'État d'Israël n'est pas encore reconnu par la France. Il déclare :

14 Georges Wormser (1888-1978), normalien, agrégé des lettres, conseiller d'État, fondateur d'une banque privée qui porte son nom, est surtout connu pour son rôle auprès de Clemenceau dont il est le directeur de cabinet en 1917 puis plus tard de Georges Mandel. Membre actif du consistoire de Paris et du consistoire central, il devient président de l'association consistoriale de Paris en 1945, puis du consistoire central en1953 et le demeure jusqu'en 1960, année de sa démission. Partisan intransigeant du francojudaïsme, il développa sa vision du judaïsme français dans son ouvrage Français israélite. Une doctrine, une tradition, une époque, Paris, éditions de Minuit, 1964.

${ }^{15}$ Cf. Edmond Dreyfus, Quatre années au consistoire (1946-1949), Paris, Saint-Gilles, 1953. 
« Français avant tout, nous devons attendre de connaître les décisions de notre gouvernement ${ }^{16}$. Cependant lors de sa visite à Paris, Chaïm Weizmann, président de l'État d'Israël venu pour plaider la reconnaissance de l'État hébreu par la France, est invité à l'office du vendredi soir où la foule des fidèles lui réserve un accueil enthousiaste. Quelques jours plus tard une cérémonie d'hommage aux soldats israéliens tombés pendant la guerre d'Indépendance est organisée à la synagogue de La Victoire.

Pendant toutes les années où il fut président du consistoire de Paris puis président du consistoire central, Georges Wormser refusa d'accorder à l'ambassadeur d'Israël une place privilégiée lors des cérémonies officielles organisées à la synagogue de La Victoire, en le plaçant parmi les autres membres du corps diplomatique accrédités à Paris, dans le strict respect des règles protocolaires. Cependant, pendant la Guerre des Six jours, à l'occasion d'une cérémonie à la synagogue de La Victoire pour le salut d'Israël, l'ambassadeur d'Israël est placé au premier rang, à gauche, aux côtés du représentant de la ville de Paris, les ambassadeurs des autres nations se trouvant dans les rangées suivantes. Ce protocole est désormais strictement observé pour toutes les cérémonies officielles à la synagogue de La Victoire. C'est là une manière de marquer la centralité d'Israël par rapport au judaïsme diasporique et la place particulière de sa représentation diplomatique pour les Juifs de France. Par ces manifestations symboliques, la synagogue de La Victoire est de plain-pied le théâtre de l'évolution doctrinale du judaïsme français marqué profondément par les lois raciales du gouvernement de Vichy et les déportations puis divisé sur son rapport à Israël.

Plus encore que par les convictions intimes de ses membres, la communauté juive de France est profondément transformée par l'arrivée progressive, à partir des années 1950, des Juifs d'Afrique du Nord. Jusqu'alors majoritairement composée d'ashkénazes, elle prend un virage à $180^{\circ}$ par l'arrivée massive de Juifs séfarades, plus pratiquants et n'ayant subi que marginalement le traumatisme des persécutions raciales de la Seconde Guerre mondiale. Depuis son ouverture en 1875 la synagogue de La Victoire est restée fidèle au rite dit « de Francfort » ou « rite alsacien » sans tentative de rapprochement avec un autre rite ${ }^{17}$. En ce sens, elle ne se distingue pas de la plupart des synagogues parisiennes et même

\footnotetext{
${ }^{16}$ Ariel Danan, « Les relations avec l'État d'Israël de 1948 à 1943 », dans Jacques Canet et Claude Nataf (dir.), op. cit, p. 334-337.

${ }^{17}$ Félix Loeb, «La Victoire ou la fidélité à un rite orthodoxe» dans Jacques Canet et Claude Nataf (dir.), op. cit, p. 452-459.
} 
hexagonales, à l'exception de la synagogue de la rue Buffault à Paris et des synagogues du sud-ouest et de Provence fidèles au rite hispanoportugais ou au rite comtadin. L'arrivée de Juifs d'Afrique du Nord a contraint plusieurs synagogues, notamment à Paris, à un aggiornamento et au remplacement du rituel en vigueur par un rituel séfarade avec plus ou moins de troubles et de difficultés.

La synagogue de La Victoire a subi la modification de la composition de la communauté juive de France. Mais elle a su intelligemment permettre le maintien des traditions liturgiques des uns et des autres en ouvrant, en son sein, deux oratoires, l'un pour le rite égyptien et l'autre pour le rite tunisien. Restant ainsi fidèle à son rôle de synagogue emblématique du judaïsme français puisqu'elle en réunit les composantes ashkénaze et séfarade et qu'elle permet le respect des liturgies de chacun. C'est ce qui a fait écrire à Pierre Brami ancien président de l'oratoire tunisien de La Victoire : « Ensemble Tunisiens, Égyptiens et Ashkenazim, nous sommes La Victoire $»^{18}$.

Cette transformation de la composition de la communauté se traduit également dans l'origine des rabbins et des ministres officiants qui se sont succédé à la synagogue de La Victoire. Jusqu'en 1963, ses rabbins ont tous été choisis au sein de l'élite du rabbinat français et étaient tous ashkénazes. En 1963, le rabbin André Chekroun, originaire d'Algérie, devient rabbin de La Victoire. Le rabbin Braka lui succède en 1990. Puis il est remplacé par le rabbin Gilles Bernheim originaire d'Alsace auquel a succédé, à nouveau, un séfarade le rabbin Moché Sebbag. De même les ministres officiants depuis 1961, bien que formés magistralement à la liturgie ashkénaze, sont d'origine séfarade comme Adolphe Attia et Aron Hayoun.

Ce rôle symbolique est encore attesté en mai 1968 lorsque La Victoire est la seule synagogue occupée par des étudiants. Ces derniers contestent la gestion de l'institution consistoriale et des organismes sociaux de la communauté mais ils expriment en même temps une critique très motivée de la forme des offices. Adversaires de la présence de l'orgue et des chœurs dans une synagogue, celle de La Victoire est selon eux le symbole de ce qu'ils rejettent ${ }^{19}$. Ce mouvement de contestation aboutit à la suppression de l'orgue dès 1968 puis, quelques années plus tard, de l'usage du microphone pour les offices de shabbat et des fêtes, à l'initiative

\footnotetext{
${ }^{18}$ Jacques Canet et Claude Nataf (dir.), op. cit, p. 352.

${ }^{19}$ Cf. Moïse Cohen, «Mai 68 et la synagogue de La Victoire », dans Jacques Canet et Claude Nataf (dir.), op. cit, p. 366-369; Claude Nataf «Le consistoire et mai 68 », Archives Juives, revue d'histoire des Juifs de France, ${ }^{\circ} 52 / 1,1^{\text {er }}$ semestre 2019, p. 111124.
} 
du grand rabbin de Paris, Alain Goldmann, malgré les protestations d'une très grande partie des fidèles.

L'histoire de la synagogue de La Victoire est donc toujours inséparable de l'histoire du judaïsme français. Elle représente bien la pluralité identitaire du judaïsme français. Lors des événements tragiques qu'a connus la communauté juive de France ces dernières années, c'est à la synagogue de La Victoire que les plus hautes autorités de l'État sont venues exprimer leur sympathie, leur soutien et la nécessité de maintenir le modèle républicain qui a permis aux Juifs de France l'intégration pleine et entière à la Nation.

Mais ce rôle symbolique de la synagogue de La Victoire, qui a été peu ou prou maintenu après le Seconde Guerre mondiale, semble aujourd'hui remis en cause. Le développement en France d'une ultraorthodoxie inspirée de courants israéliens, délaissant l'ouverture aux savoirs profanes, entraîne une critique de la synagogue de La Victoire considérée certes comme une communauté orthodoxe mais trop ouverte sur l'extérieur et principalement sur la cité. Par ailleurs, le consistoire de Paris a édifié un grand ensemble moderne dans le XVII ${ }^{\mathrm{e}}$ arrondissement abritant à la fois ses services, des salles de cours et de réunions et une immense synagogue. Les lieux ont été inaugurés par le président Emmanuel Macron en 2019. Le consistoire a tendance à y organiser un certain nombre de grandes manifestations qui se déroulaient auparavant à la synagogue de La Victoire. Il semble cependant qu'il lui sera difficile d'ôter à cette dernière son statut de lieu emblématique pour la réception des autorités publiques, des personnalités étrangères et des cérémonies retransmises par la télévision nationale.

La beauté de la synagogue, ses ornements, sa dimension, son histoire que l'on peut lire en contemplant ses murs, son entretien auquel collabore avec un soin jaloux la ville de Paris, lui donnent une mission permanente que les contradictions qu'elle peut recéler ou entraîner ne semblent pas pouvoir estomper. 


\section{La synagogue La Victoire}

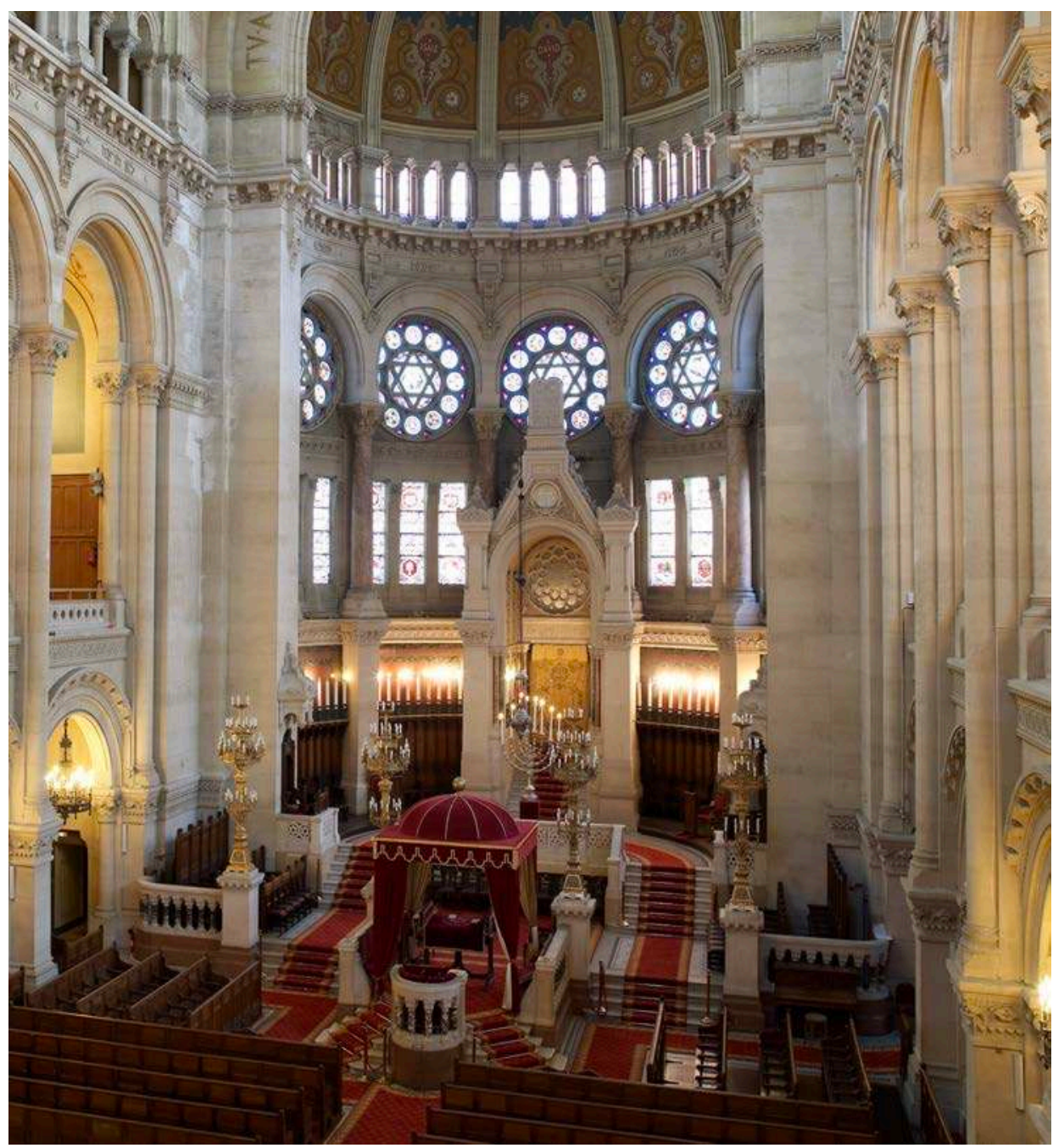

Vue plongeante sur l'estrade (bema) derrière laquelle se trouve l'armoire sainte (aron haqodesh). Les symboles juifs sont nombreux : les étoiles à six branches dans les vitraux, les tables de la Loi surplombant l'armoire sainte, les chandeliers.

Le volume de l'ensemble donne une idée de l'importance de l'édifice. La perspective illustre bien que le modèle suivi est celui des cathédrales.

Nous adressons nos remerciements à M. Jacques Canet, président de la synagogue La Victoire, pour nous avoir transmis toutes les photos de cette page et des suivantes et de nous avoir autorisés à les publier. 
La synagogue La Victoire : lieu de mémoire

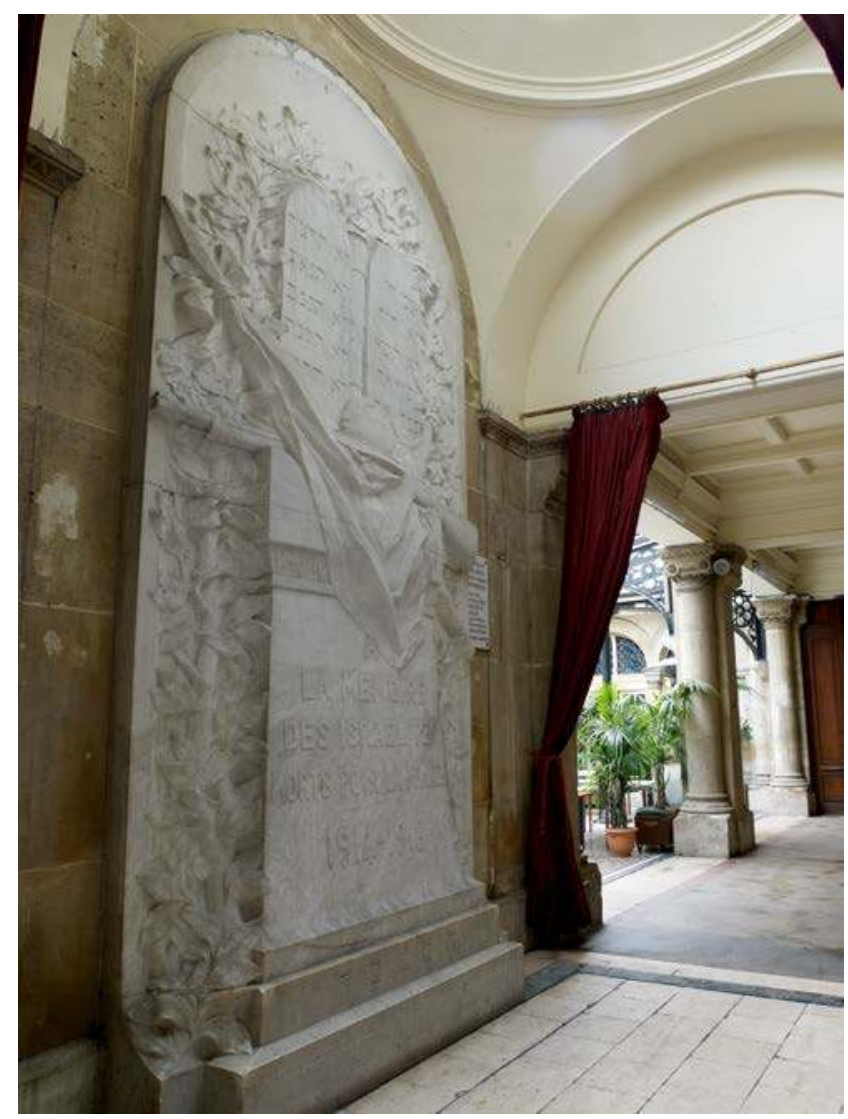

Monument en hommage aux combattants juifs morts au Champ d'honneur pendant la Première Guerre mondiale.

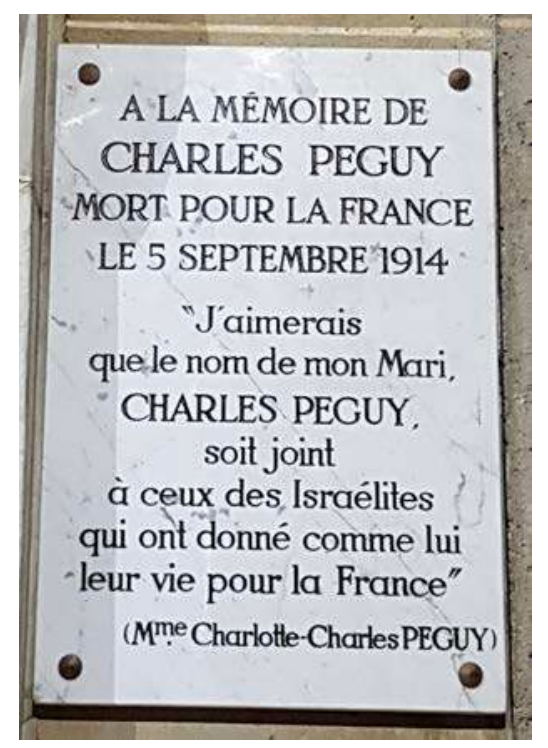

Plaque à la mémoire de Charles Péguy qui s'engagea parmi les premiers pour la défense de Dreyfus et compta, parmi ses amis, des juifs comme l'historien Jules Isaac, Bernard Lazare, Léon Blum.

Il est décédé lors des premiers combats de la guerre, le 5 septembre 1914 .

La plaque est apposée face au monument en hommage aux juifs tombés durant la Première Guerre mondiale. 


\section{La synagogue La Victoire : lieu de mémoire}

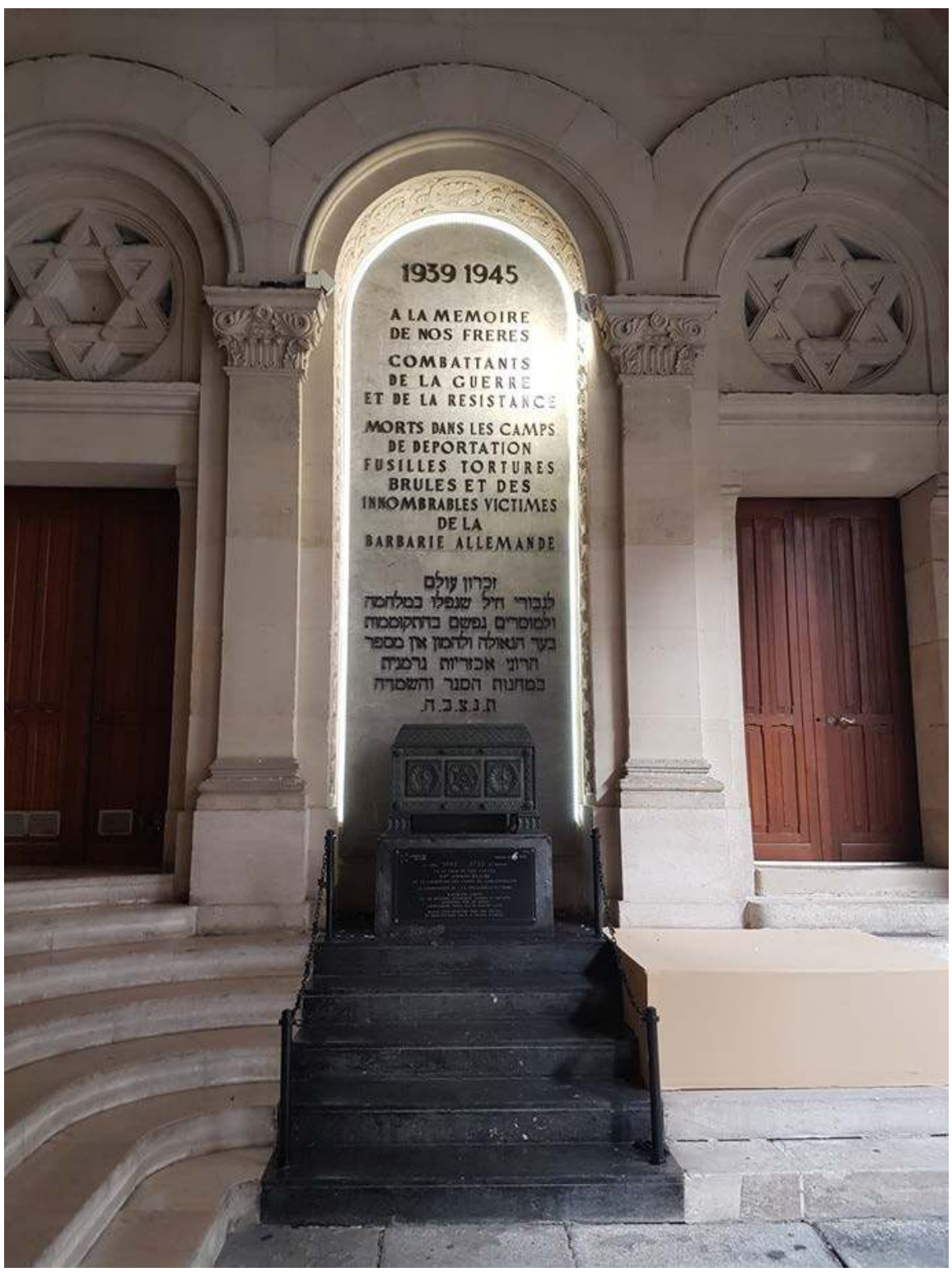

Monument, inauguré en 1949 par le président de la République Vincent Auriol, à la mémoire des Juifs morts durant la Seconde Guerre mondiale, au Champ d'honneur ainsi que dans les combats de la Résistance et en déportation. 
La synagogue La Victoire : expression d'appartenance à la Nation

Façade de la synagogue, 44 rue de la Victoire

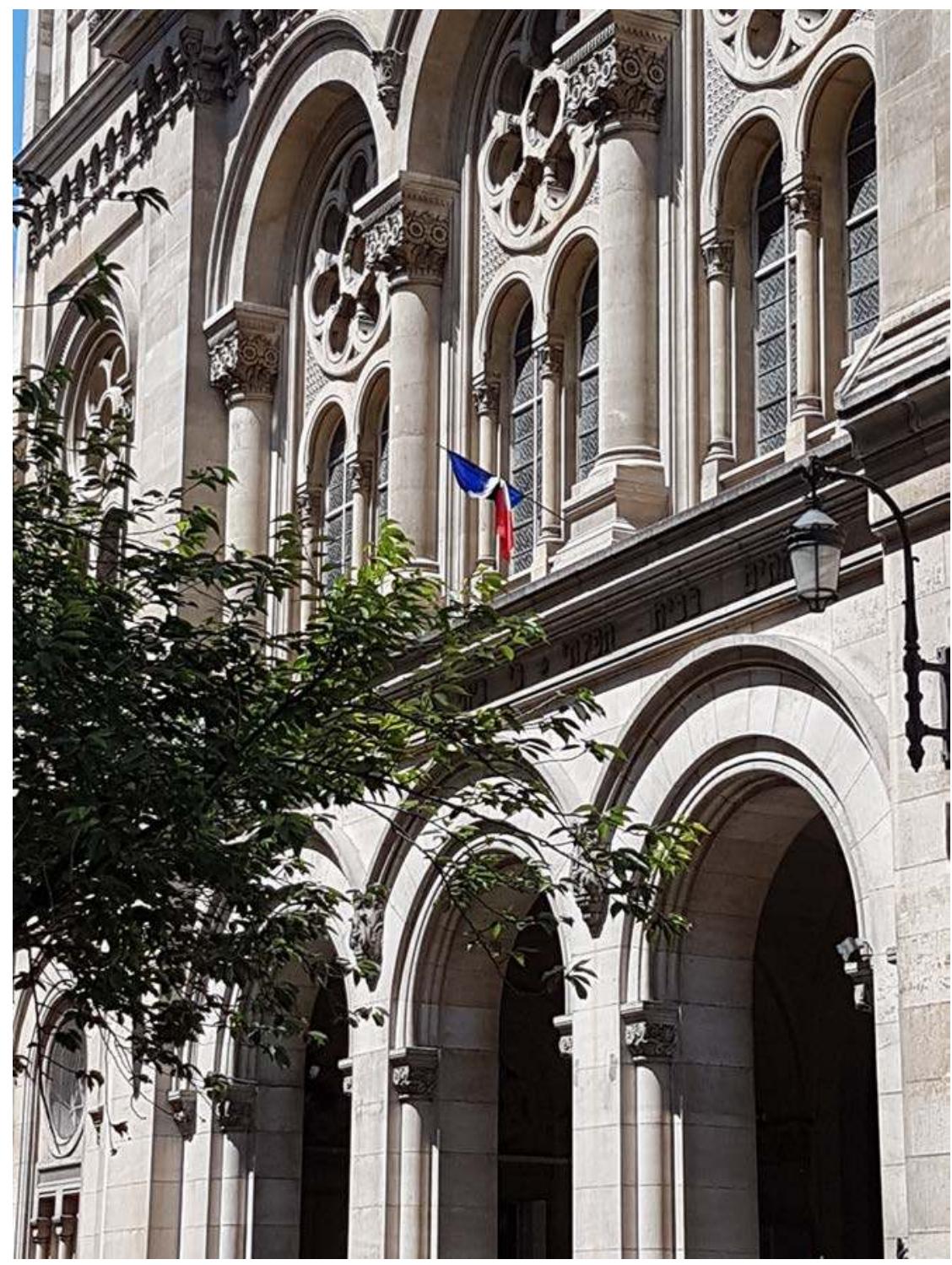

Le drapeau, placé en permanence au fronton de l'édifice, est en berne comme ceux de tous les bâtiments publics,

à la suite de l'attentat de Nice, le 14 juillet 2016. 